\title{
The Effect of Polyvinyl Alcohol Addition on the Physico-Chemical Properties of ZnO Synthesised by Ethylene Glycol-Hydrothermal Method
}

\begin{abstract}
The effect of the addition of very small amount of polyvinylalcohol (PVA) on the resulting physicochemical properties of $\mathrm{ZnO}$ synthesized by ethyleneglycol-hydrothermalmethod was studied. Significant change in particle size distribution and specific surface area can be observed even only $0.02 \%$ PVA (w/w) was added into the ethyleneglycol (EG) solution, in which the median particle size distribution increased from 5 to $8 \mathrm{~nm}$ and at the same time the specific surface area is also increased to nearly 50\%. Further addition of PVA resulted in the decrease of the specific surface area due to the formation of agglomeration and aggregation of particles as observed by FESEM study. This study shows that the presence of minute amount of polymer, PVA plays very crucial role in determining the resulting properties of the synthesized $\mathrm{ZnO}$ by EG-hydrothermalmethod.
\end{abstract}

Keyword: ZnO; Particle size distribution; Glycol-hydrothermal method 